\title{
Structural Dynamics in Biology: A Bridge Given by Implicit Vibratory Crossed Models
}

\author{
Yves Gourinat ${ }^{1}$, Laura Christon ${ }^{2}$, Frédéric Lachaud ${ }^{1}$ \\ ${ }^{1}$ ISAE-SUPAERO Université de Toulouse, Toulouse, France \\ ${ }^{2}$ ITIC Université Paul-Valéry Montpellier, Montpellier, France \\ Email: yves.gourinat@isae-supaero.fr, laura.christon@etu.univ-montp3.fr
}

How to cite this paper: Gourinat, Y., Christon, L. and Lachaud, F. (2021) Structural Dynamics in Biology: A Bridge Given by Implicit Vibratory Crossed Models. Engineering, 13, 237-256.

https://doi.org/10.4236/eng.2021.135018

Received: February 14, 2021

Accepted: May 28, 2021

Published: May 31, 2021

Copyright (c) 2021 by author(s) and Scientific Research Publishing Inc. This work is licensed under the Creative Commons Attribution International License (CC BY 4.0).

http://creativecommons.org/licenses/by/4.0/

(c) (i) Open Access

\begin{abstract}
This article proposes a synthesis and contribution at three levels: generation of dynamic equations of shell structures interacting with fluids, reduction of implicit resolution, and cross-applications to aerospace tanks and living systems. The synthesis of the equations is proposed around the four principles of thermodynamics at the level of discrete, structural and digitized systems. The implicit approach envisages an innovative analysis in terms of condensation and digitization, with in particular a perspective towards singular and integral methods. Some illustrations are proposed, in the field of performed research models and also in the fields of educational applications in biodynamics. The proposed bridge links, on one hand, the analytical Lagrange-Feynman's approach, and on the other hand experimental results obtained in laboratory and numerical experiments obtained with multiphysics software. Finally, the realized models concern conservative and dissipative models for the active and passive control of complex systems, in a unified approach.
\end{abstract}

\section{Keywords}

Vibrations, Fluid Shell Interaction, General Dynamics, Implicit Dynamics, Structural Entropy, Biophysics

\section{Introduction}

Living cells are complex, controlled and dynamic systems. They are governed by the laws of the universe, i.e. physics, and therefore fundamentally by the four principles of thermodynamics. The development, life and death of cells are comparable to their counterparts in structures, especially thin shells interacting with fluids.

The purpose of this paper is to present in parallel, in a synoptic way, the re- 
versible and irreversible phenomena observed at the cellular, structural and organic scales (see [1] [2]). The physical tools presented in particular can be considered as a common language linking all these systems. Several forays into information theory make it possible to specify the evolutions, in particular on entropic phenomena.

In terms of observability and controllability, complex systems give rise to a variety of models which-in the end-are all based on the four principles of thermodynamics. This approach has made it possible to model several biological systems, including vestibular elements, the basilar membrane, the organ of Corti, the cerebrospinal system in its traumatological approach (see [2] [3]) and makes it possible to envisage openings in both the dynamic nature of the surrounding structures and in biodynamics. The goal is here to provide with a global presentation of the present opportunities for unified analytical dynamics formulation and performed applications to fluid-solid systems, including living cells.

\section{Potential Structural Models and Results}

\subsection{Implicit Potential Model}

The simplest dynamic modeling consists of declining the first two principles-based on the two state functions temperature and internal energy-according to exchanges between kinetic and potential energies. Basically, this amounts to expressing Newton's equivalence principle digitally in matrix form, via the Lagrange-Hamilton equations:

$$
m \overrightarrow{\boldsymbol{O P}}^{\circ}-\overrightarrow{\boldsymbol{f}} \equiv \overrightarrow{\boldsymbol{O}} \Rightarrow\left[M_{i j}\right]\left(q_{j}\right)^{\circ}+\left[K_{i j}\right]\left(q_{j}\right) \equiv(O) \quad \text { (Feynman) }
$$

where

m: punctual mass (particle);

$O$ : affine Galilean origin (euclidian);

$P$ : point associated with $\mathrm{m}$ (trajectory);

$\circ$ : particle derivative with respect to time;

$\vec{f}$ force associated with $m$;

$\left(q_{j}\right)$ : column vector of $\mathrm{n}$ Lagrange displacement parameters $(j=1, \cdots, n \in N)$;

$\left[M_{i j}\right]: n \times n$ matrix of system inertias, associated with the $q_{j}$ parameters $(i, j=1, \cdots, n)$;

$\left[K_{i j}\right]: n \times n$ matrix of system stiffnesses, associated with the $q_{j}$ parameters.

To be more precise, the central arrow of Equation (1) contains all the dynamic principles, whose synoptic presentation could be summarized as shown hereunder; it can be observed that the structure of all these equations is similar, as shown hereunder.

- First step: Equivalent enthalpy

Equivalence (closed system)

$$
\overrightarrow{\boldsymbol{v}}^{\circ}-\overrightarrow{\boldsymbol{g}} \equiv \overrightarrow{\mathbf{0}}
$$

(Newton) 1st principle 
Dynamics (digital system)

$$
\left\langle\mathcal{L}_{j}\right\rangle\left(E_{\text {lag }}\right)-Q_{j d y n} \equiv Q_{j s t a t}
$$

(Lagrange) 4th principle,

$$
\text { with }\left\langle\mathcal{L}_{j}\right\rangle \equiv \frac{\mathrm{d}}{\mathrm{d} t}\left(\frac{\partial}{\partial q_{j}^{\circ}}\right)-\frac{\partial}{\partial q_{j}}
$$

- Second step: Kinetic exchanges \& exergy

$$
\begin{array}{lcc}
\text { Continuous } 1 D & U^{\circ}-c_{N}^{2} U^{\prime \prime} \equiv 0 \text { with } c_{N}^{2} \equiv \frac{E}{\rho} \quad \text { (Mach-Hooke) } \\
\text { Continuous } 1.5-2.5 D & \rho S V^{\circ \circ}+E I V^{\prime \prime \prime \prime} \equiv 0 ; \rho h W^{\circ}+D \Delta \Delta W \equiv 0 \\
& & \text { (Mach-Hooke) } \\
\text { Discrete 3D } & h^{2} \Delta \psi+8 \pi^{2} m E_{\text {ham }} \psi \equiv 0 & \text { (Schrödinger) }
\end{array}
$$

Feynman's equivalence formalism is valid in the linear domain with defined $[K]$ and $[M]$ matrices, but remains applicable in the non-linear domain with living matrices. Thus, the fundamental approach works step by step.

To be complete, we can observe that Feynman's equivalence is based on the four principles, by truncature at resonance. In fact, the complete equation, shown here in the general formalism of Lagrange and in the linear digital frame, is in correspondance with the principles as proposed hereunder:

$$
\begin{gathered}
\left\langle\mathcal{L}_{j}\right\rangle\left(E_{\text {kin }}\right)-\left\langle\mathcal{L}_{j}\right\rangle\left(E_{\text {opt }}\right) \equiv Q_{\text {jfeyn }}+Q_{\text {jstat }} \\
{[M](q)^{\circ}+[K](q) \equiv(Q)_{\text {feyn }}+(Q)_{\text {static loads }}} \\
\{\operatorname{Pr} 2 ; \quad \operatorname{Pr} 1\} \quad\{\operatorname{Pr} 3 ; \quad \operatorname{Pr} 4\}
\end{gathered}
$$

where $Q_{j f e y n} \equiv Q_{j d y n p o t}+Q_{j} \quad$ (dynamic loads \& dissipation).

It should be noted at this level that Maxwell-Betti's reciprocity theorem implies the symmetry of these matrices (constant or not) and the principle of inertia the fact that they are real positive semi-definite matrices. They will even be defined positive $\left(m_{i i}>0, k_{i i}>0\right)$ in the case of structures which admit a static reference configuration.

However, eigenmodes can be explained algebraically in the linear domain because the whole system can be resolved on a harmonic basis. Indeed, the diagonalization allows to make an equivalence of the whole dynamics with systems represented by a single Degree of Freedom (DoF).

Thus, it is possible to define an implicit analytical impedance matrix, in which the time has explicitly disappeared:

$$
\begin{aligned}
& {\left[Z_{i j}\right] \equiv-\omega^{2}\left[M_{i j}\right]+\left[K_{i j}\right] ; i, j=1, \cdots, n} \\
& \Rightarrow\left[Z_{i j}\right]\left(q_{j}\right) \equiv(0) \Rightarrow-\omega_{i}^{2} \mu_{i}+\chi_{i} \equiv 0
\end{aligned}
$$

where $\left[Z_{i j}\right]$ structural harmonic impedance matrix of the system, associated with the $q_{j}$ parameters.

$\omega \in \mathbb{R}^{+*}:$ system pulsation ( $\mathrm{rd} / \mathrm{s}$ with classical Lagrange parameters);

$\omega_{i} \in \mathbb{R}^{+*}$ : eigenpulsation of the system (natural or circular pulsation);

$\left(\mu_{i}, \chi_{i}\right) \in \mathbb{R}^{+* 2}$ : effective modal inertia \& stiffness of the system. 
This approach is the basis for all classical dynamic models, including biodynamic systems. In particular, it allows tracing a first genome of the systems with linear tools (see [4] [5] [6] [7] [8]).

Of particular note are the important possibilities for thin structures (beams and shells) in vibroacoustic interaction with their fluid environment, which lend themselves very well to simplectic and numerical analytical models, thus providing an efficient modal representation and a powerful tool for first approach theoretical observability and controllability.

It is the fundamental tool for topological design support, initial dynamic diagnosis and Generalized Predictive Control (see [9] [10] [11] [12]) with passive \& active controls. And even for topological optimization.

The advantage of the implicit approach is to include time in the harmonic response; it is also its weak point.

The first two principles of thermodynamics respond perfectly one to another, and it is therefore an excellent classical model. It covers perfectly the classical motion of an electron around a proton, the vibrations of a Lagrange beam, or the classical Maxwell equations in the Minkowski-Einstein Euclidean universe. Constitutively, however, it is euclidian and does not assume the history of the system.

Even if it can be solved explicitly (Newmark's scheme) this system of representation is thus intrinsically adapted to an implicit resolution, the Kramer-Gauss modal one. However, the latter has been most powerful in the representation by truncated modal bases in cyclic dynamics and by shock spectra in transient dynamics. It can thus be very useful to represent the severity of a dynamic environment in fatigue and structural seismology (see [13] [14] [15]). But the principle of causality cannot be represented since it does not appear in the axioms of this representation.

It does not mean that the causality does not exist, but that it is only implicit. But the implicit time is not the time of the history of the structure, it is simply the number of cycles, or the implied frequencies, but fundamentally the system is built around 2 base units of length and mass ( $\mathrm{m}$ and $\mathrm{kg}$ in ISO system, see Figure 1) with time being implicit. And this system can last until explicit time arrives.

\subsection{Classical Entropic Structural Model}

If we wish to go further, we must introduce the other two principles of thermodynamics, which are inseparable from one another (as are the first two). The following example will be used in the linear framework, but the principle extends to the general case.

We therefore introduce an explicit function of time which is neither inertia nor generalized potential, and which is sometimes called excitation, but which we will call external force and which will be represented canonically-according to Fourier and Kronecker-by an elementary harmonic function. 


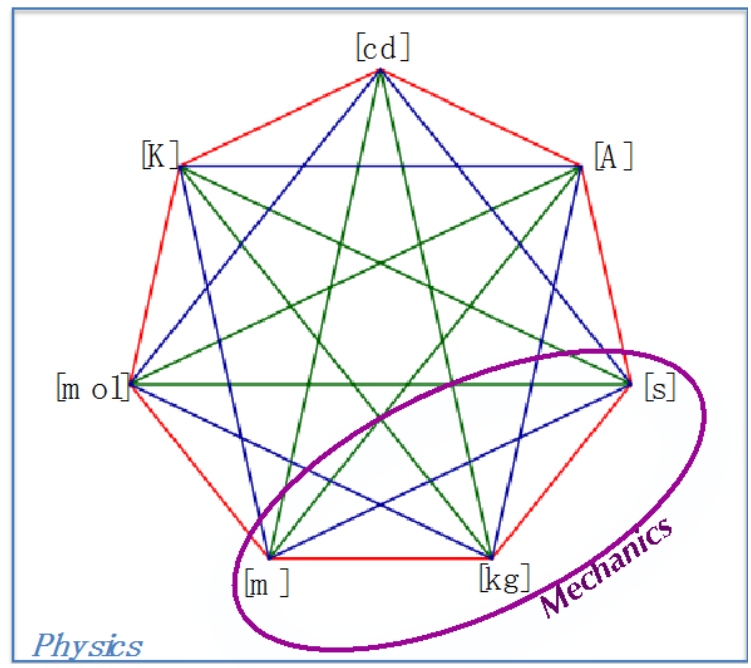

Figure 1. The mechanical domain.

This induces an explicit response (called particular solution of the complete equation) emphasizing the dynamic amplification factor \& resonance:

$$
\mu p_{e}^{\circ}+\chi p_{e} \equiv \cos \left(\omega_{e} t\right) \Rightarrow p_{e} \equiv \beta_{e} \cos \left(\omega_{e} t\right) \Rightarrow \alpha_{e} \equiv \chi \beta_{e}=\frac{1}{1-\gamma_{e}^{2}}
$$

where $(\mu, \chi) \in \mathbb{R}^{+* 2}$ modal inertia and stiffness;

$p_{e}(t) \in \mathbb{R}$ main parameter in the canonical response to canonical $\cos \left(\omega_{e} t\right)$ external dynamic load;

$\beta_{e} \in \mathbb{R}$ amplitude of the canonical response;

$\alpha_{e} \in \mathbb{R}$ dynamic amplification ratio (DAR);

$\gamma_{e} \equiv \frac{\omega_{e}}{\omega} \in \mathbb{R}^{+*}$ dimensionless external pulsation (frequency or angular rate) $\left(\omega_{e} \in \mathbb{R}^{+*}\right)$.

The $\alpha_{e}$ DAR factor introduces causality into the model, but in a particular solution. It raises the concept of resonance, which will require an additional (fourth) term of dissipation. This makes it possible to envisage explicit models incorporating structural damage.

In linear models, the dissipation is the viscosity (solid and fluid) but as this is only very approximate in a solid (and in a non-Newtonian fluid) we will rather introduce an equivalent dimensionless viscous damping (integrating all the dissipations-linear or not-around the resonance) which truncates the amplitudes (or operates a frequency shift) in the vicinity of the resonances.

The vicinity of the resonances is moreover precisely defined by the bandwidth: Frequency amplitude-resonance bandwidth

$$
\left\{\begin{array}{l}
\gamma_{e} \in[1-\zeta ; 1+\zeta] \subset \mathbb{R} \\
\alpha_{e \max }= \pm \frac{1}{2 \zeta}
\end{array}\right.
$$

where $\zeta$ dimensionless dissipation; for structures $\zeta \cong 0.01$;

$\alpha_{e \max }$ dynamic quality factor or resonance surtension ratio. 
The $\zeta$ factor from which $\alpha_{e \max }$ is derived integrates the nonconformities of the structure; it should be noted that it depends-in non-linear models-on absolute frequency values, and must therefore be associated with each resonance. In an n-mode model, this set is therefore represented by then $\zeta_{i}$ values $(i=1, \cdots, n)$ which define a truncation or frequency slip envelope that contains non-conformity or system damage information.

It should be noted that this entropic representation had been proposed by Feynman in the linear framework, indicating that at resonance the terms viscous and excitation cancel each other out, leaving the two Newtonian terms free. In other words, at resonance, the external force-by definition resonance-compensates the dissipation, and maintains the perpetual motion of the perfect model. This observation-extremely pertinent-is generalized to all dynamic systems thanks to dissipation envelopes.

It applies as much in observability as in modal controllability (see [16] [17]). It should be observed that these related terms (external force and excitation) are actually induced by the arrival of the $3 \mathrm{rd}$ and 4 th principles of thermodynamics in the model, namely the explicit function of time which proceeds from the history of the system, and the dissipation which reflects its internal damage, thus its intrinsic state.

Then we have a complete mechanical model, which can be declined quite simply to thin solid elements (straight beams, curved beams, plates, shells) in their Newtonian interaction (incompressible perfect fluid, compressible perfect fluid, Newtonian fluid). They have given rise to advanced structural modelling involving solid-fluid metamodes (see Figure 2).

The models have been performed with COMSOL Multiphysics ${ }^{\circledast}$ software with the shell elements, and with Newtonian and non-newtonian fluids. This numerical approach has been performed both for the cryotechnic tank and the organic systems; the aim here is precisely to show that this kind of model, with similar equations, can be applied to both kinds of systems.

Indeed, the general matrix model applies as well to Lagrangian displacements in the case of a solid as to Eulerian pressure fluctuations in the case of a fluid, which makes it possible to offer hybrid models combining, for example, very
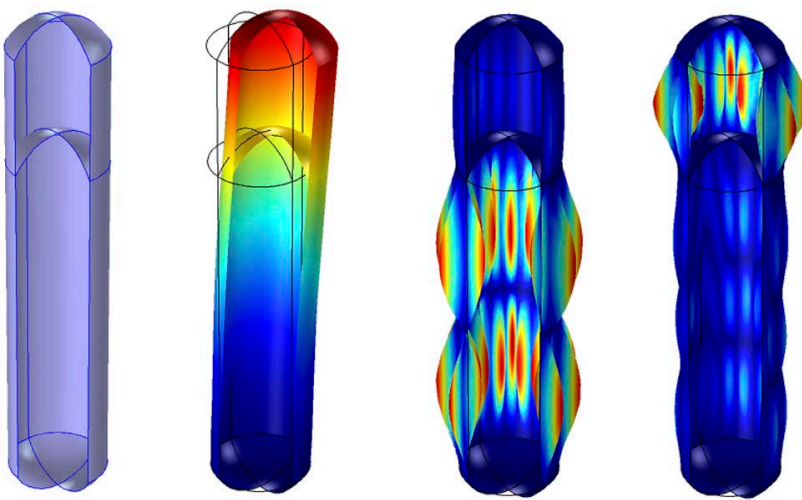

Figure 2. Solid-fluid metamodes (cryotechnic tank). 
simply shells and perfect fluids (see [18]).

These models are transposable to living systems such as a cell (membrane with fluid interaction) or an organic structure: shell in interaction with soft materials, and in a specific environment. For instance, in biodynamics, the tonopic coupling in the cochlea, with the basila membrane, is modelised by the plate-fluid theory (Figure 3).

Last but not least, in the dissipation itself, a linear term of viscous damping can be distinguished from a contribution related to other frictions, both in solids and fluids. In solids, the non-linear part represents precisely the damage or defects (delaminations, cracks, discontinuities) and in the fluid, the realization of tests with equivalent granular fluids in similarity to Froude makes it possible to observe this type of irreversibility (see Figure 4).

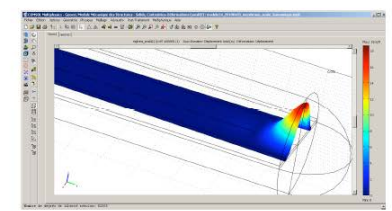

Mode $1(87 \mathrm{~Hz})$

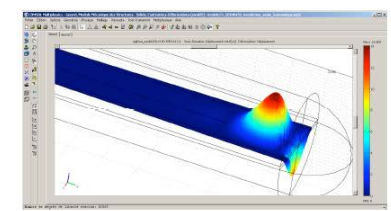

Mode $2(100 \mathrm{~Hz})$

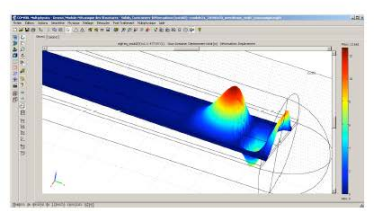

Mode $3(111 \mathrm{~Hz})$

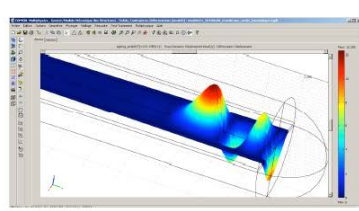

Mode $4(122 \mathrm{~Hz})$

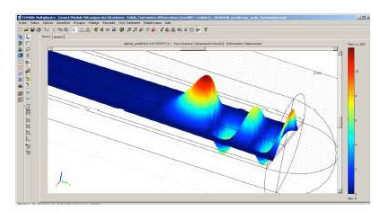

Mode $5(132 \mathrm{~Hz})$

Figure 3. Basilar membrane tonotopic model.

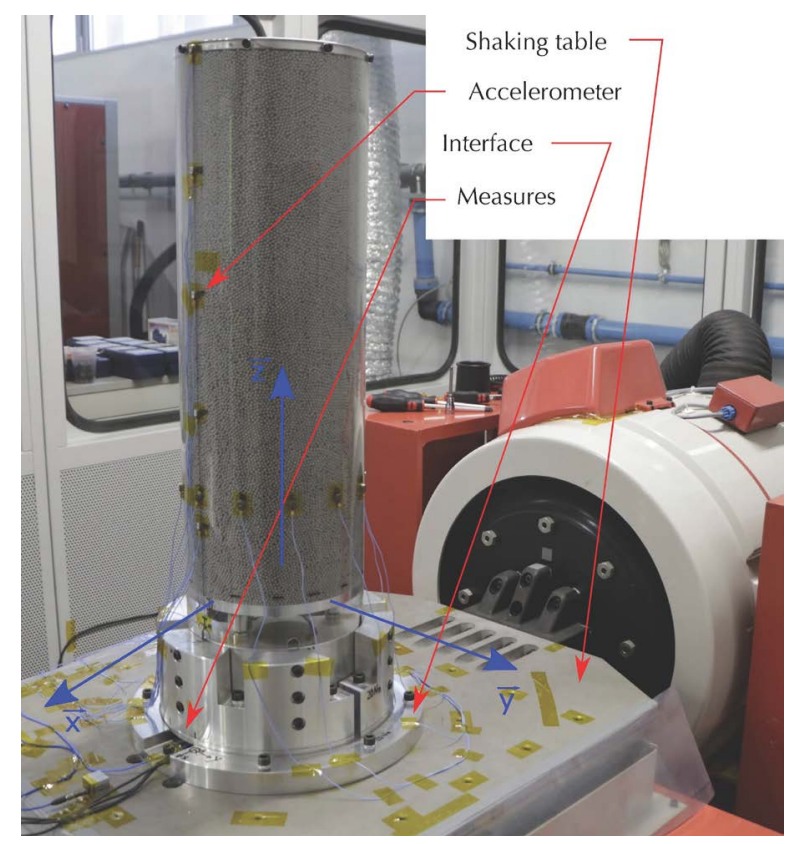

Figure 4. Cryotechnic tank test with granular material for identification of metamodes. 


\subsection{General Model for Passive and Active Control}

On the basis of the resonance zone-delimited by dissipation in the probabilistic sense-both observability and controllability of the models can be fairly easily shown. Indeed, this zone of unknowingness (in the Heisenberg sense) is the intersection between an observable and non-controllable past and a non-observable \& controllable future. And what guarantees this possibility is precisely that it is based on principles three and four, and that there are in fact conservations that address both observable kinetic quantities (Routh conservations) and controllable energy quantities (Hamilton-Painlevé conservation).

This synthesis intrinsically covers the non-linear domain (see [19]) and indeed, analogies begin to appear between these equations of dynamics (the shells and dissipation in particular) and those of general gravitation. Potentially, it applies to all interactions, thus involving the seven basic quantities of physics.

Remaining in the field of solid-fluid mechanics, the set of the 4 terms of dynamic equations thus constitutes an important potential source that can feed both cellular simulators in their interactions and organic simulators (see [20]). This transfer can be performed both on continuous or discrete analytical approaches, and on digitized models according to the three main families of digitization (finite elements, particle domains, integral methods). Not only the dynamic behavior is modelled, but also the history of the complete system in its environment. The representation is thus both an observation \& control tool, allowing to carry out the finest virtual experiments, and modelling in particular not only the entropic evolution of the structure (3rd principle) but also its reference potential configuration (4th principle).

On the basis of the resonance zone-delimited by dissipation in the probabilistic sense-both observability and controllability of the models can be fairly easily shown. Indeed, this zone of unknowingness (in the Heisenberg sense) is the intersection between an observable and non-controllable past and a non-observable and controllable future. And what guarantees this possibility is precisely that it is based on principles three and four, and that there are in fact conservations that address both observable kinetic quantities (Routh conservations) and controllable energy quantities (Hamilton-Painlevé conservation).

\section{Cellular Shell-Fluid Representation}

\subsection{Lagrange-Euler Frame, the Plate-Fluid Model}

The lagrangian bended plate moves with the $2.5 \mathrm{D}$ classical equation, given by principles 2 and 1 , inducing $W$ displacement along $Z$ ( $X, Y, t$ field):

$$
D \Delta \Delta W+\rho h W^{\circ \circ} \equiv p_{Z}
$$

$$
\begin{aligned}
& D \equiv \frac{E h^{3}}{12\left(1-v^{2}\right)} \in \mathbb{R}^{+*} \text { lineic bending stiffness; } \\
& (\rho, h) \in \mathbb{R}^{+* 2} \text { platevolumic mass \& thickness; } \\
& p_{Z}(X, Y, t) \in \mathbb{R} \text { external normal surface static load applied on the plate. }
\end{aligned}
$$


It is pertinent to observe that this equation is similar to the prestressed membrane law. This is also the case for the $1 \mathrm{D}$ rod equation, similar to the rope law.

In parallel, we could consider a perfect compressible fluid in a tank, precisely in a rigid tank (galilean solid reference) closed by a flexible plate (see Figure 5).

This demonstrator is the typical flexible structure for the basic hybrid dynamic representation:

1) $W(X, Y, t)$ lagrangian relative dynamic $Z$-displacement $2 \mathrm{D}$ field in the plate/membrane;

2) $p(X, Y, Z, t)$ eulerian pressure fluctuation $3 \mathrm{D}$ field in the perfect fluid.

In the fluid, the dynamic behaviour is given by the Euler's equation (Navier-Stokes 2 without viscous stress):

$$
(\rho \overrightarrow{\boldsymbol{V}})^{\circ}+\overrightarrow{\operatorname{div}}(\rho \overrightarrow{\boldsymbol{V}} \otimes \overrightarrow{\boldsymbol{V}}+p \overline{\overline{\boldsymbol{I}}}) \equiv \overrightarrow{\mathbf{0}}
$$

$\otimes$ dyadic product

completed with the continuity condition (N-S 1):

$$
\rho^{\circ}+\operatorname{div}(\rho \overrightarrow{\boldsymbol{V}}) \equiv 0
$$

Thus, Equations (5) to (7) drive the motion of the system (plate or fluid).

We observe that implicitly in the linear modal frame, the Euler-Helmholtz equations in the (perfect) fluid can be written as:

$$
\left\{\begin{array}{l}
a^{2} \Delta p-\omega_{i}^{2} p=0 \text { in } \Omega \\
\partial_{Z} p-\rho_{f} \omega_{i}^{2} W=0 \text { on } \partial \Omega \\
\iiint_{\Omega(\text { fluid })} p \mathrm{~d} X \mathrm{~d} Y \mathrm{~d} Z+\iint_{\partial \Omega(\text { plate })} \rho_{f} a^{2} W \mathrm{~d} X \mathrm{~d} Y=0 \text { (Stokes resultant) }
\end{array}\right.
$$

$(a, \rho, p) \in \mathbb{R}^{+* 3}$ fluid sound celerity, volumic mass, eulerian pressure fluctuation;

$\Omega \subset \mathbb{R}^{3}$ volumic fluid domain;

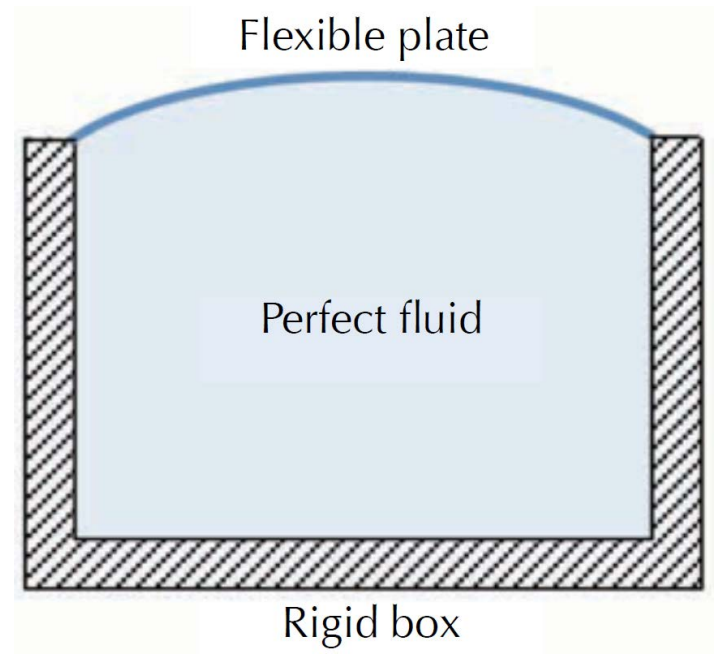

Figure 5. Elementary plate-fluid configuration (drum model). 
$\partial \Omega \subset \mathbb{R}^{2}$ surfacic plate interface (plate surface).

It is pertinent to observe that this equation is similar to the prestressed membrane law. This is also the case for the 1D rod equation, similar to the rope law. Thus, Equations (5) to (7) drive the motion of the system (plate or fluid).

\subsection{Discretized Shell-Fluid System-Digital Modes}

Then, the 2D plate will be discretized, as well as the $3 \mathrm{D}$ fluid volume. At each node of the system will be attached: a Lagrangian $\mathrm{W}$ displacement at each node of the shell (plate) and an Eulerian $p$ pressure fluctuation at each node of the (perfect) fluid. In total, we have nodes: $n_{s}$ structural (solid) nodes in the plate and $n_{f}$ nodes in the fluid.

$$
n=n_{s}+n_{f}
$$

The dynamic digital modes of the system will be valid for these $n$ hybrid Degrees of Freedom (DoF).

At this level-and in order to reinforce the full mechanical pertinence of these hybrid modes-it is useful to observe that the Mach-Hooke equation previously mentioned in a rod has exactly the same expression in displacement and in stress:

$$
U^{\circ}-c_{N}^{2} U^{\prime \prime}=0 \Leftrightarrow \sigma_{X X}^{\circ}-c_{N}^{2} \sigma_{X X}^{\prime \prime}=0
$$

The reason is simply the rheological relation between $\sigma_{X X}$ tension/compression stress and $U$ deflection in a rod:

$$
\sigma_{X X}=E \varepsilon_{X X}=E U^{\prime}
$$

This allows to represent the global dynamic system (plate + fluid) with $\mathrm{n}$ hybrid DoFs: $n_{s}$ W Lagrangian normal deflections and $n_{f}$ pressure Eulerian fluctuations.

Thus, $n_{s}$ is the number of nodes in the plate, and $n_{f}$ the number of nodes in the fluid. The hybrid impedance matrix becomes natural to model the motion of the system:

$$
\begin{aligned}
& {\left[Z_{i j}\left(\omega_{k}^{2}\right)\right]\left(r_{j}\right) \equiv\left(0_{j}\right), i, j, k=1, \cdots, n}
\end{aligned}
$$

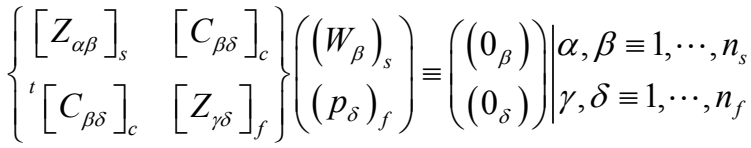

with $\left[Z_{\alpha \beta}\right]_{s}$ solid $n_{s} \times n_{s}$ structural impedance matrix of the solid plate $2 \mathrm{D}$ domain;

$\left[Z_{\gamma \delta}\right]_{f}$ fluid $n_{f} \times n_{f}$ acoustic (Helmholtz) impedance matrix of the fluid 3Ddomain;

$\left[C_{\beta \delta}\right]_{c}$ coupling impedance $n_{s} \times n_{f}$ matrix structure-fluid 2.5D domain;

$\left(W_{\beta}\right)$ plate structural $n_{s}$ vector of W lagrangian deflections (plate DoFs);

$\left(p_{\delta}\right)_{f}$ fluid $n_{f}$ vector of eulerian pressure fluctuations (Helmholtz DoFs).

By the principles 1 and 2 of thermodynamics (Galilean reversibility \& Maxwell's reciprocity) all $[Z]$ matrices are symmetrical, real positive semi-definite in 
classical physics.

In addition, as the fluid is here considered perfect, the Helmholtz matrix is diagonal in any base (for any paving/meshing of the fluid).

Thus, for the considered mesh/pavage, the information contained in the $[Z]$ matrices are summarized as follows:

1) the $[Z]_{\mathcal{S}}$ solid/plate matrix is defined by its upper $n_{s} \times n_{s}$ triangle:

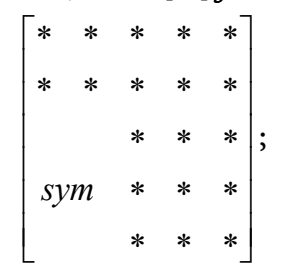

2) it is thus defined by $n_{s}+\left(n_{s}-1\right)+\left(n_{s}-2\right)+\cdots+1=n_{s}\left(n_{s}+1\right) / 2$ independent scalar numbers;

3) the $[Z]_{f}$ fluid matrix is $n_{f}$ diagonal $\left[\begin{array}{lllll}* & 0 & 0 & 0 & 0 \\ * & * & 0 & 0 & 0 \\ & * & 0 & 0 \\ s y m & * & * & 0 \\ & * & * & *\end{array}\right]$ representable by the $n_{f}$ vector: $\left(\begin{array}{c}H_{1} \\ H_{1} \\ \vdots \\ H_{n_{f}}\end{array}\right)$;

4 ) it is thus defined by $n_{f}$ independent scalar numbers,

5) then the $[C]_{s f}$ solid-fluid coupling matrix is a full $n_{s} \times n_{f}$ one;

6) it is thus defined by $n_{s} \times n_{f}$ independent scalar numbers.

Consequently, the global $\{Z\}$ matrix $(n \times n)$ which models the complete plate-fluid dynamics, is represented by $\left(n_{s}+1\right) n_{s} / 2+n_{f}+n_{s} n_{f}=\left(n_{s}+1\right)\left(2 n_{s}+n_{f}\right) / 2$ scalar numbers. That is the implicit dynamic representation of the global system, valid for any method of digitization (FEM, DEM, BEM-F, D, B).

This preliminary approach can be extended to large \& complex systems, for which $n_{s} \& n_{f} \gg 1$. For this purpose, let's consider that the plate (which represents a generic thin structure) is linked with a cubic volume. With these assumptions, if the mesh is coherent between the fluid and the solid: $n_{f} \sim n_{s}^{3 / 2}$. The previous expression of representative scalar numbers is then depending only on the structural representation $\left(n_{s}+1\right)\left(2 n_{s}+n_{s}^{3 / 2}\right) / 2 \sim 0.5 n_{s}^{5 / 2}$.

To conclude this approach of the digitization of a shell-perfect fluid $n_{s} \times n_{f}$ structure, the expression of the $\mathcal{N}_{D o F}$ number of scalars required (necessary and sufficient) for a complete implicit digital representation-i.e. the number of digital hybrid Degrees of Freedom-is given by:

$$
2 \mathcal{N}_{\text {DoF }}=\left(n_{s}+1\right)\left(2 n_{s}+n_{f}\right) ; 2 \overline{\mathcal{N}_{\text {DoF }}} \sim n_{s}^{5 / 2}
$$

That is the implicit dynamic representation of the global system. The applica- 
tion field is here the domain of thin shells with gaseous or ultra-light fluid (LH2).

\subsection{Condensation}

If we consider an optimized/local mesh, focusing consequently on a FEM-type model, the matrices are not only symmetrical, but also hollowed. With standard quadrangular/hexaedral elements, the number of representative scalar numbers is reduced to $8 n_{s}+24 n_{f}+12 n_{s}=8\left(3 n_{s}+4 n_{f}\right)$.

In explicit solution, the corresponding $[M]$ and $[K]$ matrices are well adapted to Cholesky's direct inversion.

Thus, the standard FEM cost in terms of scalar number (memory size for $\mathrm{Z} / \mathrm{M} / \mathrm{K}$ matrices) is given by:

$$
\mathcal{S}_{F E M}=8\left(3 n_{s}+4 n_{f}\right) ; \overline{\mathcal{S}_{F E M}} \sim 32 n_{s}^{5 / 2}
$$

This figure is valid to size the memory and power of the digital process, and is thus relevant in terms of internal (for computation) representation of the global system, whereas the $\mathcal{N}_{D o F}$ number represents the physical external approach.

The symmetrical impedance matrix, including the structural and fluid dynamics, gives implicitly—by diagonalization-the eigenvalues \& eigenshapes, Lagrange-Euler metamodes (solid + fluid). That is the modal dynamic representation of the global system. Inventoriating the information in the complete external (meta-)modal base, we obtain:

1) $n \omega_{i}$ eigenvalues (pulsations);

2) $n\left(p_{j}\right)_{i}$ eigenvectors (of $W$ or $p$ amplitudes for each mode).

Each eigenvector is defined with a multiplicative constant, and so represented by $n-1$ independent scalars. Thus, the whole modal base requires $n+n(n-1)=n^{2}$ independent scalars. That is the complete dynamic external signature (or "DNA") of the solid-fluid system.

$$
2 \mathcal{N}_{\text {mod }}=n^{2} \equiv\left(n_{s}+n_{f}\right)^{2} ; 2 \overline{\mathcal{N}_{\text {mod }}} \sim n_{s}^{3}
$$

In dynamic engineering, it is often possible to truncate the base. A performing strategy consists of considering the three first modes (the three lowest frequencies) or the three first ones plus a fourth representative of a specific phenomenon (optional local mode or sensitive mode, considering the external loads).

For a well-conditioned system, this approach allows a precision better than $1 \%$. Consequently, the truncated dynamic model of the global system will be represented by $4 n$ independent scalars, which is a very simple representation, the basic dynamic DNA of the system.

$$
2 \mathcal{N}_{\text {trunc } 4}=8 n \equiv 8\left(n_{s}+n_{f}\right) ; 2 \overline{\mathcal{N}_{\text {trunc } 4}} \sim 8 n_{s}^{3 / 2}
$$

Through the boundary-integral methods, it is allowed to represent a plate (2D) with a mesh on its 1D frontier, and the volumic fluid by its frontier with the plate. Thus, with this ultimate condensation, the $[\mathrm{M}]$ and $[\mathrm{K}]$ global matrices 
(plate + fluid) are reduced to a $n_{s} \times n_{s}$ one. These matrices are full and non-symmetrical but solvable explicitly by iterative methods. Provided that $M$ and $\mathrm{K}$ switch, then they admit a common basis for diagonalization and the implicit approach can be applied to this integral model.

\subsection{Solid-Fluid Interaction in Closed Shells}

Considering perfect fluids, the previous fluid equations are unchanged in the general case of a volumic tank, both for internal and external fluids, the $X, Y, Z$ deflection on the shell being referenced as $U, V, W$ :

$$
\delta \overrightarrow{\vec{P}} \equiv\left(\begin{array}{l}
U \\
V \\
W
\end{array}\right)_{X, Y, Z}
$$

If $n_{n}$ is the number of nodes in the shell (structural nodes) then the number of shell structural DoFs is $n_{s}=3 n_{n}$. The fluid equations are then connected with the Reissner-Naghdi shell equations where the lagrangian deflection of mid-surface is expressed in the local curvature axes (see [19] [20]). More precisely, this method solves the dynamic equivalence principle with $U, V, W, Q_{X Z}$ $Q_{Y Z}$ hybrid variables, but $U, V, V$ are actually the structural ones for the shell dynamics.

The previous system shown on the plate example remains valid, the solid mesh being on curvilinear coordinates on the shell. The unique difference is, for the external fluid only, the Stoke's resultant condition being replaced by Sommerfeld limit condition:

$$
p \rightarrow 0 \text { when }\|\overrightarrow{\boldsymbol{O P}}\| \rightarrow \infty \quad \text { (Sommerfeld) }
$$

Connected with the Reissner-Naghdi $U, V, V$ equations of shells, the previous Euler-Stokes-Sommerfeld system generates the global dynamics of the shell in a perfect fluid.

A viscous newtonian fluid requires the third Navier-Stokes equation, including the shear stress. Consequently, the standard shell digital dynamic implicit matrix equation of a Reissner-Naghdi shell in a newtonian fluid keeps the shape defined in (13); the shapes of the implied block-matrices are as follows:

$$
\begin{gathered}
{\left[Z_{\alpha \beta}\right]_{s}=\left[\begin{array}{ccccc}
Z_{11} & Z_{12} & * & 0 & 0 \\
* & Z_{22} & * & * & 0 \\
& & * & * & * \\
& s y m & & * & * \\
& & & & Z_{n_{s} n_{s}}
\end{array}\right] ;} \\
{\left[Z_{\gamma \delta}\right]_{f}=\left[\begin{array}{ccccc}
H_{11} & S_{12} & S_{13} & * & 0 \\
& H_{22} & S_{23} & * & 0 \\
& & * & * & * \\
& s y m & & * & S_{\left(n_{f}-1\right) n_{f}} \\
& & & & H_{n_{f} n_{f}}
\end{array}\right] ;}
\end{gathered}
$$




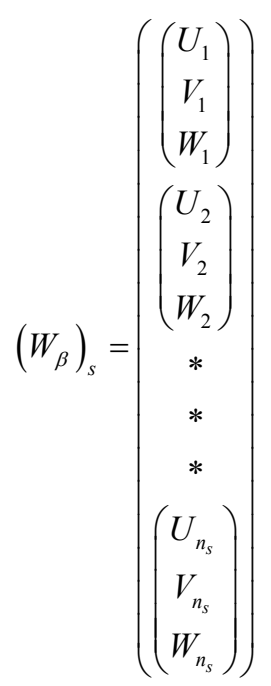

The field application is here thick shells in viscous fluids, such as hydraulic systems or submarine skins, but these models can also be combined with the previous ones.

To determine the level of the model (viscous/non-viscous, shell/membrane, static/dynamic) it is necessary to examine several adimensional numbers, considering the dimensions and characteristics of the tank (Figure 6).

For the fluid model, it is possible to consider Stoke's equation valid for Newtonian fluids:

$$
\begin{gathered}
\rho \frac{\partial \overrightarrow{\boldsymbol{V}}}{\partial t}+\rho(\overrightarrow{\boldsymbol{V}} \cdot \overrightarrow{\boldsymbol{g r a d}}) \overrightarrow{\boldsymbol{V}}+\overrightarrow{\boldsymbol{g r a d}} p-\mu \Delta \overrightarrow{\boldsymbol{V}}-\left(\mu^{\prime}+\frac{\mu}{3}\right) \overrightarrow{\boldsymbol{g r a d}}(\operatorname{div} \overrightarrow{\boldsymbol{V}})-\rho \overrightarrow{\boldsymbol{f}}_{\text {vol }} \equiv \overrightarrow{\mathbf{0}} \\
\overline{\overline{\boldsymbol{\Sigma}}}_{f} \equiv 2 \mu \overline{\overline{\mathcal{E}}}_{f}^{\circ}+\mu^{\prime} \operatorname{tr}\left(\overline{\overline{\mathcal{E}}}_{f}^{\circ}\right) \overline{\overline{\mathbb{I}}}
\end{gathered}
$$

where $\vec{V}$ Eulerian velocity in the fluid;

$\mu, \mu^{\prime}$ primary \& secondary Newtonian viscosities of the fluid; $\overline{\bar{\Sigma}}_{f}, \overline{\overline{\mathcal{E}}}_{f}^{\circ}$ stress \& strain-rate tensors in the fluid;

$\overline{\bar{I}}$ unitary Kronecker's tensor.

It is thus possible to observe different adimensional numbers:

1) Acoustic Reynolds Number $\operatorname{Re}_{a c} \equiv \frac{\omega_{c} \mathcal{L}_{c}^{2}}{v_{c}} \mid v_{c} \quad$ kinematic viscosity $\left(v_{c} \equiv \frac{\mu}{\rho}\right)$

High: model with perfect fluid. Low. model with viscous fluid.

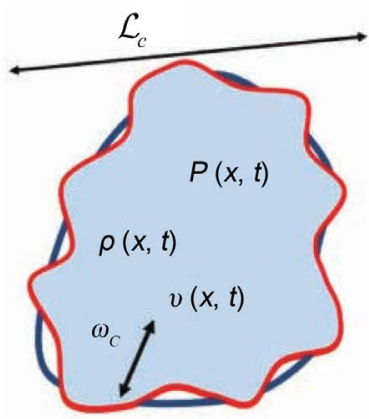

p eulerian pressure

$\rho$ volumic mass

$\checkmark$ eulerian velocity

$\omega_{c}$ circular frequency

$\mathcal{L}_{\mathrm{c}}$ typical cell length

Figure 6. Typical monophasic cellular tank. 
2) Oscillatory Froude Number $F r_{\text {osc }} \equiv \frac{\mathcal{L}_{c} \omega_{c}^{2}}{g} \mid g$ equivalent gravity, for static loads.

High: dynamics independent from statics. Low. coupled model.

3) Flow Detachment Number $F d_{s} \equiv \frac{V_{t}}{\omega_{c} R_{e x t}}$ tangential velocity on the shell; $R_{\text {ext }}$ shell curvature radius, on external normal.

High: turbulent model. Low. laminar Coanda model.

4) Weber's Number $W e_{f} \equiv \frac{\rho \omega_{c} \mathcal{L}_{c}^{3}}{\sigma} \mid \sigma$ surface tension in the fluid.

High: surface tension neglected. Low. with surface tension.

Important parameter for multiphasic systems.

\subsection{Models for Complex Systems \& Cells}

The topology, geometry and singularity of the structure and its control-model can be used as an indicator of its entropy. That approach is also an occasion to mention the modern methods (in progress) whose potentiality open wide perspectives in the domain of complex structure thermodynamics and, consequently, in the field of cellular and organic modeling.

In comparison with FEM and DEM, the Boundary Elements Method (BEM) is the most promizing one for such combined problems. In the case of an uncompressible fluid, the Equation (6) is reduced to anholonomic condition between the boundary (plate) DoFs. In the general case of the shell with a newtonian fluid, the condensation leads to a ns dimension problem. In a certain way, it is equivalent to condensate the behavior of the cellular tank to its boundary represented by the shell. The global BEM can be used for multiphasic 3D structures, with $2 \mathrm{D}$ external and internal interfaces (see [21]). These interfaces-regular C0 varieties-can have local (discrete) or smooth (extended) singularities. These local singularities can be classified as C1 (angular), C1 (sharp) or C2.5 (angular \& crack). The smooth singularities represent a diffusion, such as porosity (Figure 7).

In the context of BEM, particular boundary elements can be developed, in similarity with Robinson's finite element for a crack (see [22] [23]). The BEM considering the mesh only on the interface surfaces ( $2 \mathrm{D}$ variety) this allows a reduced numerical model. And this model is directly based on the four principles, as all the exchanges cross these surfaces.

But we can offer to go further. Indeed, the shell itself can be meshed according to its interfaces, which are reduced to the singular discrete lines between panels (see [24] [25]). If we consider the canonical example of the plate interacting with a perfect fluid (drum membrane), this amounts to meshing the plate only along its perimeter, which constitutes a $1 \mathrm{D}$ variety.

Thus, a cubic shell will be meshed by its arches, which is an important advance in terms of digital condensation. In this perspective a complex assembly of 


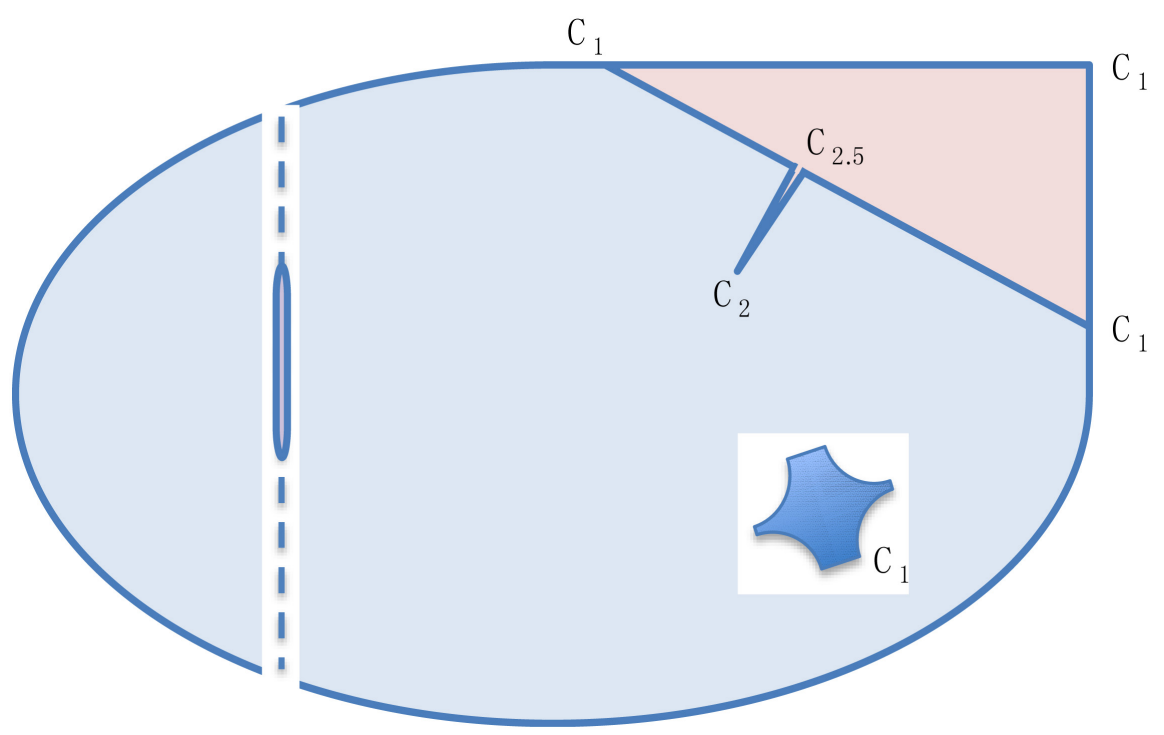

Figure 7. Interfaces and singularities in a complex shell.

shells will be represented by the intersection lines, or even the points (nodes) interfacing these lines. We could thus obtain a very concentrated thermodynamic model of the structural cell. Its implicit resolution gives metamodes, and its explicit integration allows to explore transient evolutions. And the ratio between the explicit and implicit results could even constitute an indicator of the entropy of the controlled system.

\section{Conclusions}

The methods presented in this article bring together achieved and present work on current aerospace structures and research on innovative methods in two directions. Vibratory dynamics thus open tracks that integrate both the modeling of structures and the modal representation of information.

The first explored direction concerns active and passive control: Generalized Predictive Control (see [10] [11] [14]) and Operational Modal Diagnosis (see [16] [17]). These issues can be expressed in terms of controllability and observability of complex systems. Through the approach based on the four principles of thermodynamics, this paper proposes an opening towards the reversibility of processes, leading for example to the modal set of plans for dynamic structures. For this purpose, and to demonstrate the perspective for any physical system, the Appendix proposed a synoptic presentation of dynamic equations in relation with the principles.

The second direction concerns the optimal hybrid modeling of thin structures coupled with Newtonian fluids. The proposed synthesis, in the presented structuration of the equations, integrates fluid-solid meta-modes in Gauss' sense, as well as proposals for condensation by integral methods with perspectives on the two-levels condensed representation: by boundary conditions and modal extraction, leading to vibratory optimal compression of models. 


\section{Acknowledgements}

Jean-Emmanuel Chambe, Miguel Charlotte, Jean-Emmanuel Alibert, Matthieu Hospital, Yulia Akisheva; ISAE-SUPAERO. Vincent Lapoujade, Charlotte Michel, Tess Legaud, Thomas Pierrot, Antoine Guilpin; DynaS+. ValiaFascio, Mathieu Delorme; Ateca.

\section{Conflicts of Interest}

The authors declare no conflicts of interest regarding the publication of this paper.

\section{References}

[1] Selva, P., et al. (2010) Toward a Three-Dimensional Finite-Element Model of the Human Inner Ear Angular Accelerometers Sensors. International Journal for Computational Vision \& Biomechanics, 3, 149-156.

[2] Foucaud, S., et al. (2014) Artificial Cochlea \& Acoustic Black Hole Traveling Waves Observation: Model \& Experimental Results. Journal of Sound \& Vibration, 333, 3428-3439. https://doi.org/10.1016/j.jsv.2014.03.016

[3] Cournoyer, J. and Hoshizaki, T.B. (2019) Head Dynamic Response and Brain Tissue Deformation for Boxing Punches with and without Loss of Consciousness. Clinical Biomechanics, 67, 96-101. https://doi.org/10.1016/j.clinbiomech.2019.05.003

[4] Shahdin, S., et al. (2011) Evaluation of the Impact Resistance of Various Composite Sandwich Beams by Vibration Tests. Shock \& Vibration, 18, 789-805. https://doi.org/10.1155/2011/259295

[5] Shahdin, A., et al. (2011) Correlating Low Energy Impact Damage with Changes in Modal Parameters: Diagnosis Tools \& FE Validation. Structural Health Monitoring, 10, 199-217. https://doi.org/10.1177/1475921710373297

[6] Cornette, D., Kerdreux, B., Michon, G. and Gourinat, Y. (2015) Aeroelastic Tailoring of Helicopter Blades. Journal of Computational \& Nonlinear Dynamics (ASME), 10, Article ID: 061001. https://doi.org/10.1115/1.4027717

[7] Feng, D., et al. (2016) Vision Based Multipoint Displacement Measurement for Structural Health Monitoring. Structural Control Health Monitoring, 23, 876-890. https://doi.org/10.1002/stc.1819

[8] Qing, X., Li, W.Z., Wang, Y.S. and Sun, H. (2019) Piezoelectric Transducer-Based Structural Health Monitoring for Aircraft Applications. Sensors, 19, 545. https://doi.org/10.3390/s19030545

[9] Niemann, H., Morlier, J., Shahdin, A. and Gourinat, Y. (2010) Damage Localization Using Experimental Modal Parameters \& Topology Optimization. Mechanical Systems \& Signal Processing, 24, 636-652. https://doi.org/10.1016/j.ymssp.2009.10.022

[10] Mueller, J., et al. (2006) A Numerical Study on Active Control for Tiltrotor Whirl Flutter Stability Augmentation. Journal of the American Helicopter Society, 51, 244-254. https://doi.org/10.4050/1.3092885

[11] Liu, X., Zhang, C.H., Li, K. and Zhang, Q. (2017) Robust Current Control-Based Generalized Predictive Control with Sliding Mode Disturbance Compensation for PMSM Drives. ISA Transactions, 71, 542-552.

https://doi.org/10.1016/j.isatra.2017.08.015

[12] Jing, G., Gao, H.F. and Xiang, J.W. (2020) A Level-Set Topological Optimization 
Method to Analyze Two-Dimensional Thermal Problem Using BEM. Applied Mathematical Modelling, 78, 37-56. https://doi.org/10.1016/j.apm.2019.10.002

[13] Maillet, I., et al. (2013) A New Test Methodology Based on Structural Resonance for Mode I Fatigue Delamination Growth in a Unidirectional Composite. Composite Structures, 97, 353-362. https://doi.org/10.1016/j.compstruct.2012.10.024

[14] Wang, X., Reysett, A., Pommier-Budinger, V. and Gourinat, Y. (2014) A Modified Preisach Model \& Its Inversion for Hysteresis Compensation in Piezoelectric Actuators. Multidiscipline Modeling Materials \& Structures, 10, 122-142. https://doi.org/10.1108/MMMS-01-2013-0004

[15] Liu, J., et al. (2019) Intelligent Time-Domain Parameters Matching for Shock Response Spectrum and Its Experimental Validation in Active Vibration Control Systems. Shock \& Vibration, 2019, Article ID: 2920845.

https://doi.org/10.1155/2019/2920845

[16] Gourinat, Y. and Belloeil, V. (2007) A Truncated Low Approach of Intrinsic Linear \& Nonlinear Damping in Thin Structures. Journal of Vibrations \& Acoustics, 129, 32-38. https://doi.org/10.1115/1.2358153

[17] Nikishkov, Y., Seon, G. and Makeev, A. (2019) Progressive Fatigue Damage Simulation in Laminated Composites Based on Explicit Finite Element Formulation. Journal of the American Helicopter Society, 64, 1-12. https://doi.org/10.4050/JAHS.64.022004

[18] Nguyen, S.K., et al. (2019) Experimental Testing of Pre-Stressed Granular Assemblies as a Surrogate Material for the Dynamic Analysis of Launcher Cryogenic Tanks. Engineering Structures (Elsevier), 197, 1-14. https://doi.org/10.1016/j.engstruct.2019.109433

[19] Nguyen, S.K., et al. (2018) Towards an Analytical Formulation for Fluid Structure Tank Vibration Analysis: Modal Equivalence Using Granular Materials. Engineering Structures, 177, 345-356. https://doi.org/10.1016/j.engstruct.2018.09.077

[20] Gourinat, Y. (2011) Essai sur les Équations Constitutives de la Dynamique des Structures. $83 \mathrm{p}$.

[21] Vellas, M., et al. (2017) AeroAging-A New Collaboration between Life Sciences Experts \& Aerospace Engineers. The Journal of Nutrition, Health \& Aging, 21, 1024-1030. https://doi.org/10.1007/s12603-017-0969-1

[22] Fernandes, G.R. and Konda, D.H. (2008) A BEM Formulation Based on Reissner's Theory to Perform Simple Bending Analysis of Plates Reinforced by Rectangular Beams. Computational Mechanics, 42, 671-683.

https://doi.org/10.1007/s00466-008-0266-2

[23] Mukhopadhyay, N.K., Maiti, S.K. and Kakodkar, A. (2000) A Review of SIF Evaluation and Modelling of Singularities in BEM. Computational Mechanics, 25, 358-375. https://doi.org/10.1007/s004660050483

[24] Haddad, M., Gourinat, Y. and Charlotte, M. (2011) Equivalence Theory Applied to Anisotropic Thin Plates. Engineering, 317, 669-679.

https://doi.org/10.4236/eng.2011.37080

[25] Gourinat, Y. (2019) La Théorie des Singularités. Un complément aux modèles locaux par éléments finis en Physique. Chapter ref.AF5070, Éditions Techniques de l'Ingénieur. 


\section{Appendix: The Four Principles in Fourteen Equations}

Below is proposed a sequential and synoptic presentation of fourteen dynamic equations with comparable $2+2$ term structures.

Thus is highlighted the explicit relation with the four principles of thermodynamics, and thus open the possibility of both implicit and explicit resolutions.

\section{Mechanics}

$\overrightarrow{\boldsymbol{V}}^{\circ}-\overrightarrow{\boldsymbol{g}} \equiv \overrightarrow{\mathbf{0}} ; \boldsymbol{m} \overrightarrow{\boldsymbol{O P}}^{\circ}-\overrightarrow{\boldsymbol{f}} \equiv \overrightarrow{\mathbf{0}} \Rightarrow[M](q)^{\circ}+[K](q)=(0)$

(Newton-Feynman, pr. 1-2) (1.1)

Kinetic constant $\frac{\partial E_{\text {lag }}}{\partial q_{k}} \equiv 0 \Rightarrow \frac{\mathrm{d}}{\mathrm{d} t}\left(\frac{\partial E_{\text {lag }}}{\partial q_{k}^{\circ}}\right) \equiv 0$

(Lagrange-Routh, pr. 2) (1.2)

Control energy $\frac{\partial E_{\text {lag }}}{\partial t} \equiv 0 \Rightarrow \frac{\mathrm{d}}{\mathrm{d} t}\left(E_{\text {ham }}-\sum_{j} \frac{\partial E_{\text {pot }}}{\partial q_{j}^{\circ}} q_{j}^{\circ}\right) \equiv 0$

(Hamilton-Painlevé, pr. 3) (1.3)

Dynamic digital system $\langle\mathcal{L}\rangle_{j}\left(E_{\text {lag }}\right)-Q_{j \text { feyn }} \equiv Q_{j \text { stat }}$

(Lagrange-Feynman, pr. 1-4) (1.4)

Structures

Continuous $1 D$ solid $U^{\circ}-c_{N}^{2} U^{\prime \prime} \equiv 0, c_{N}^{2} \equiv \frac{E}{\rho}$

(Hooke-Mach, pr. 1-2) (2.1)

Continuous $1.5 D$ solid $\rho S V^{\circ 0}+E I V^{\prime \prime \prime \prime} \equiv \lambda_{\text {Ystat }}$

(Bresse-Lagrange, pr. 1-2) (2.2)

Continuous $2.5 D$ euclidian $\rho h W^{\circ}+D \Delta \Delta W \equiv p_{\text {Zstat }}, \quad D \equiv \frac{E h^{3}}{12\left(1-v^{2}\right)}$

(Kirchoff-Lagrange, pr. 1-2) (2.3)

Cont. 2.5D non-euclidian $\mathcal{D}_{X, Y, Z}-\mathcal{F}_{X, Y, Z}=p_{X, Y, Z s t a t} ; \mathcal{T}_{Y, X}+Q_{Y Z, X Z}=0$

(Reissner-Mindlin, pr. 1-3) (2.4)

Solid $3 D \rho \overrightarrow{\boldsymbol{v}}^{\circ}-\overrightarrow{\operatorname{div}} \overline{\overline{\boldsymbol{\Sigma}}}-\overrightarrow{\boldsymbol{f}}_{\text {voldyn }}=\overrightarrow{\boldsymbol{f}}_{\text {volstat }}$

(Navier-Lamé, pr. 1-4) (2.5)

Physics

$$
\text { Newtonian fluid }\left\{\begin{array}{l}
\rho^{\circ}+\overrightarrow{\operatorname{div}}(\rho \overrightarrow{\boldsymbol{v}})=0 \\
(\rho \overrightarrow{\boldsymbol{v}})^{\circ}+\overline{\overline{\operatorname{grad}}}(\rho \overrightarrow{\boldsymbol{v}}) \cdot \overrightarrow{\boldsymbol{v}}+\rho \overrightarrow{\boldsymbol{v}} \operatorname{div} \overrightarrow{\boldsymbol{v}}-\overrightarrow{\operatorname{div}} \overline{\bar{\Sigma}}-\overrightarrow{\boldsymbol{f}}_{v o l}=\overrightarrow{\boldsymbol{O}} \\
\left(\rho e_{t o t}\right)^{\circ}+\operatorname{div}\left(\rho \boldsymbol{e}_{t o t} \overrightarrow{\boldsymbol{v}}-\overline{\bar{\Sigma}} \overrightarrow{\boldsymbol{v}}-\lambda_{T} \overrightarrow{\operatorname{grad} T}\right)-\overrightarrow{\boldsymbol{f}}_{v o l} \cdot \overrightarrow{\boldsymbol{v}}=q_{v o l}
\end{array}\right.
$$

(Navier-Stokes pr. 1-3) (3.1)

$$
\text { Newtonian gas }\left\{\begin{array}{l}
e_{g} \equiv c_{v} T \\
h \equiv e_{g}+\frac{p}{\rho} \equiv c_{p} T \\
T d s_{g} \equiv d e_{g}+p d\left(\frac{1}{\rho}\right)
\end{array}\right.
$$

(Joule-Thomson pr. 1-3) (3.2)

Mechanical discrete $3 D 8 \pi^{2} m E_{\text {ham }} \psi+h^{2} \Delta \psi \equiv 0$ 
(Schrödinger pr. 2, 1) (3.3)

Discrete $3 D$ half-spin $2 \pi c_{0}\left(m c_{0}\left[\alpha_{0}\right]+\overrightarrow{\boldsymbol{\alpha}} \cdot \overrightarrow{\boldsymbol{p}}\right) \psi \equiv i h \psi^{\circ}$

(Dirac-Schrödinger pr. 3, 2, 1) (3.4)

General continuous $4.5 D 8 \pi G_{0} \overline{\overline{\boldsymbol{T}}}-c_{0}^{4} \overline{\overline{\boldsymbol{R}}} \equiv c_{0}^{4}\left(\Lambda_{c}-\frac{\operatorname{tr} \overline{\overline{\boldsymbol{R}}}}{2}\right) \overline{\overline{\boldsymbol{\mu}}}$

(Einstein-Hilbert pr. 2, 1, 4, 3) (3.5) 